\title{
BMJ Open Impairments, activity limitations and participation restrictions experienced in the first year following a critical illness: protocol for a systematic review
}

\author{
Patricia J Ohtake, ${ }^{1}$ Jacqueline Coffey Scott, ${ }^{2}$ Rana S Hinman, ${ }^{3}$ Alan Chong Lee, ${ }^{4}$ \\ James M Smith ${ }^{5}$
}

To cite: Ohtake PJ, Coffey Scott J, Hinman RS, et al. Impairments, activity limitations and participation restrictions experienced in the first year following a critical illness: protocol for a systematic review. BMJ Open 2017;7:e013847.

doi:10.1136/bmjopen-2016013847

- Prepublication history and additional material is available. To view please visit the journal (http://dx.doi.org/ 10.1136/bmjopen-2016013847).

Received 10 August 2016 Revised 19 October 2016 Accepted 16 December 2016

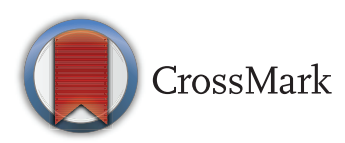

For numbered affiliations see end of article.

Correspondence to

Dr Patricia J Ohtake; ohtake@ buffalo.edu

\section{ABSTRACT}

Introduction: Critical illness requiring intensive care unit (ICU) management is a life-altering event with $\sim 25 \%$ of ICU survivors experiencing persistent reductions in physical functioning, impairments in mental health, cognitive dysfunction and decreased quality of life. This constellation of problems is known as 'postintensive care syndrome' (PICS) and may persist for months and/or years. The purpose of this systematic review is to identify the scope and magnitude of physical problems associated with PICS during the first year after discharge from ICU, using the International Classification of Functioning, Disability and Health framework to elucidate the impairments of body functions and structures, activity limitations and participation restrictions.

Methods and analysis: Medline (Ovid), Cochrane Database of Systematic Reviews (Ovid), Cochrane Central Register of Controlled Trials (Ovid), PubMed, CINAHL (EBSCO), Web of Science and EMBASE will be systematically searched for observational studies reporting the physical impairments of body functions and structures, activity limitations and participation restrictions associated with PICS. Two reviewers will assess the articles for eligibility according to prespecified selection criteria, after which an independent reviewer will perform data extraction which will be validated by a second independent reviewer. Quality appraisal will be performed by two independent reviewers. Outcomes of the included studies will be summarised in tables and in narrative format and meta-analyses will be conducted where appropriate.

Ethics and dissemination: Formal ethical approval is not required as no primary data is collected. This systematic review will identify the scope and magnitude of physical problems associated with PICS during the first year after discharge from ICU and will be disseminated through a peer-reviewed publication and at conference meetings, to inform practice and future research on the physical problems associated with PICS.

Trial registration number: CRD42015023520.

\section{Strengths and limitations of this study}

- As the number of adults with postintensive care syndrome (PICS) is increasing, an evidencebased, cohesive identification of the scope and magnitude of the impairments in physical function is essential for clinical decision-making for patient management.

- This systematic review will use the WHO's International Classification of Functioning, Disability and Health to provide a comprehensive description of the physical problems experienced by individuals with PICS.

- Since most people with PICS are community dwelling, the findings from this systematic review have the potential to inform primary care and rehabilitation practitioners about the constellation of physical impairments associated with PICS.

\section{INTRODUCTION}

More than 5.7 million adults are admitted to intensive care units (ICUs) annually for management of critical illness. ${ }^{1}$ With advances in medical and surgical management, significant numbers of patients requiring ICU care survive and are discharged from hospital. ${ }^{2}{ }^{3}$ Over the past decade, it has been increasingly recognised that critical illness requiring ICU management is a life-altering event with $\sim 25 \%$ of ICU survivors experiencing persistent reductions in physical functioning, ${ }^{4}{ }_{5}$ impairments in mental health, ${ }^{6}$ cognitive dysfunction ${ }^{78}$ and decreased quality of life. ${ }^{9}$ This constellation of problems is known as 'postintensive care syndrome' (PICS) ${ }^{10}$ and may persist for months and/or years. ${ }^{11} 12$ Specifically, individuals with PICS experience some or all of the following:

- physical problems including reduced pulmonary functioning, ${ }^{13}$ muscular weakness, ${ }^{5}$ limited function such as walking ${ }^{14}$ and difficulty returning to work; ${ }^{15}$ 
HEALTH CONDITION

Post-intensive Care Syndrome

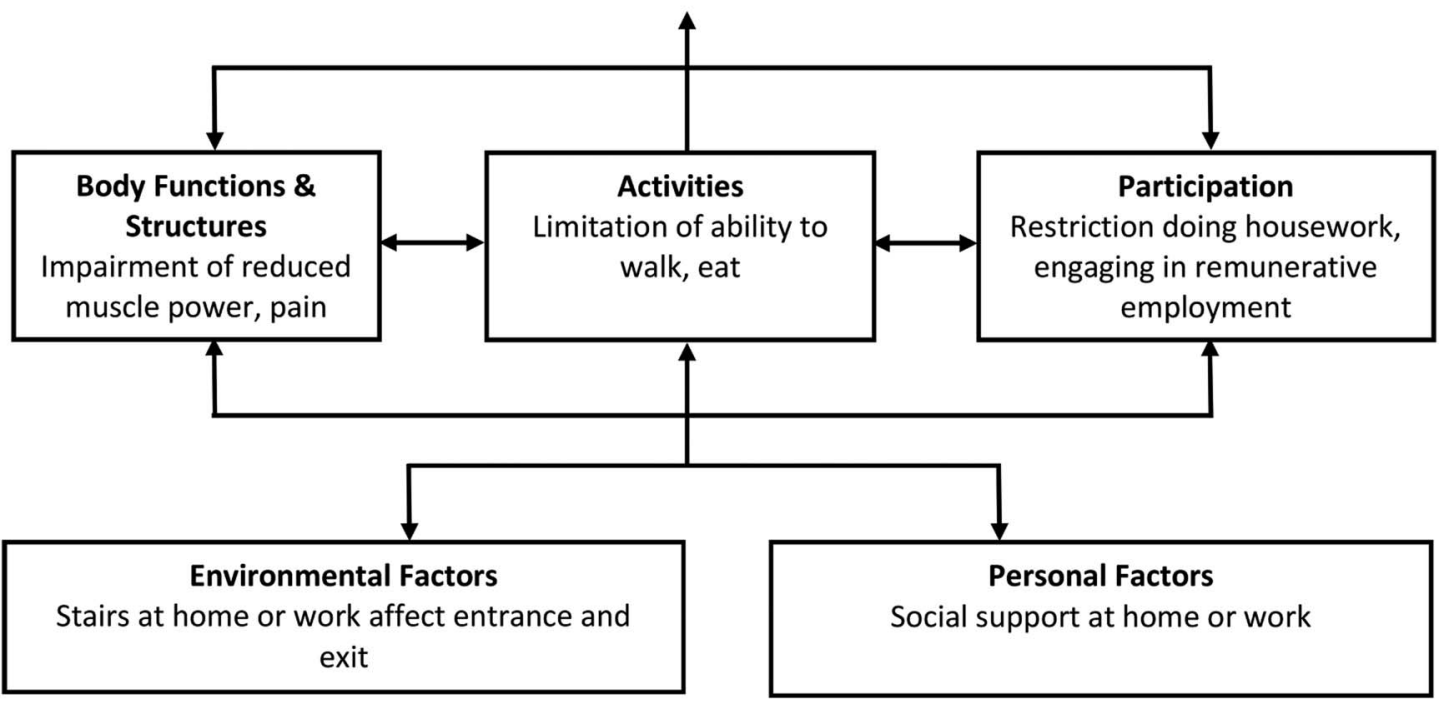

CONTEXTUAL FACTORS

Figure 1 Example of how the ICF model can be applied to PICS. ICF, International Classification of Functioning, Disability and Health; PICS, postintensive care syndrome. (Adapted from the representation of the model of disability that is the basis for ICF (p. 9), from: WHO. Towards a Common Language for Functioning, Disability and Health (ICF Beginner's Guide), 2002. Retrieved from http://www.who.int/classifications/icf/training/icfbeginnersguide.pdf. Accessed 14 June 2016).

- mental health disorders including anxiety/acute stress disorder, ${ }^{16}$ post-traumatic stress disorder ${ }^{17}$ and depression; ${ }^{18}$

- cognitive impairments, including disruption of executive function, memory and mental processing speed. ${ }^{19}$

Quality of life among survivors of intensive care, on average, is reduced relative to the general population for up to 12 years following hospital discharge. ${ }^{20}$ Recent systematic reviews found that patients who had been critically ill generally reported a lower quality of life 1 year following ICU discharge, especially in physical domains, than an age-matched and gender-matched population. ${ }^{9} 21$

With respect to the physical impairments reported in individuals following ICU discharge, many of these studies are compromised by not including the patients' premorbid physical status or a healthy comparison group, and are inconsistent in the study designs, physical outcomes reported and outcome measures used. ${ }^{15} 2223$ The inconsistent reporting is likely related to the heterogeneity of physical problems associated with PICS, as well as the diversity of outcome measures used to quantify these problems. ${ }^{22}{ }^{24}{ }^{25}$ Since the healthcare management of ICU survivors is often transferred to community-based primary care providers after hospital discharge, a clear understanding of the severity and impact of the physical problems experienced by individuals following ICU discharge is essential for appropriate diagnosis and patient management. To date, there has been no systematic review of the physical symptoms associated with PICS.
The International Classification of Functioning, Disability and Health (ICF) is the WHO's framework for measuring health and disability at individual and population levels. It is ideal for the characterisation of the breadth of physical problems associated with PICS. ${ }^{26}$ The major components of the ICF are body functions and structures (anatomy and physiology), activity (execution of a task or action by an individual) and participation (involvement in a life situation) (figure 1). ${ }^{26}$ The ICF conceptualises a person's level of functioning as a dynamic interaction between her or his health conditions, and contextual factors which include their unique environmental factors and personal situations. Once the constellation of physical impairments associated with PICS has been identified, appropriate screening paradigms using relevant outcome measures can be established to guide the rehabilitation of people with PICS and ultimately improve patient-centred health outcomes.

The purpose of this systematic review is to identify the scope and magnitude of physical problems associated with PICS during the first year after discharge from ICU, using the ICF framework to elucidate the impairments of body functions and structures, activity limitations and participation restrictions.

\section{METHODS AND ANALYSIS}

We followed the Preferred Reporting Items for Systematic review and Meta-Analysis Protocols (PRISMA-P) 2015 checklist $^{27}$ to inform and report this 
Table 1 PECOT for study inclusion

\begin{tabular}{|c|c|c|c|c|}
\hline Participants (P) & Exposure (E) & Comparison (C) & Outcomes (0) & Time (T) \\
\hline \multicolumn{5}{|l|}{ Inclusion criteria } \\
\hline $\begin{array}{l}\text { Community-dwelling } \\
\text { adults aged } \\
\geq 18 \text { years }\end{array}$ & $\begin{array}{l}\text { Medical conditions, general } \\
\text { surgical procedures, trauma } \\
\text { and burns resulting in } \\
\text { critical illness requiring } \\
\text { management in an intensive } \\
\text { care unit }\end{array}$ & $\begin{array}{l}\text { 1. Control group who did } \\
\text { not experience critical } \\
\text { illness, and/or } \\
\text { 2. Published normative } \\
\text { data for outcomes of } \\
\text { interest, and/or } \\
\text { 3. Retrospective } \\
\text { self-reports of } \\
\text { impairments, activity } \\
\text { limitations, and } \\
\text { participation restrictions } \\
\text { within a month prior to } \\
\text { developing the critical } \\
\text { illness }\end{array}$ & $\begin{array}{l}\text { 1. Physical } \\
\text { impairments } \\
\text { (problems with } \\
\text { body functions } \\
\text { and structures), } \\
\text { 2. Activity limitations } \\
\text { (problems with the } \\
\text { ability to execute } \\
\text { tasks or actions), } \\
\text { 3. Participation } \\
\text { restrictions } \\
\text { (problems with the } \\
\text { ability to be } \\
\text { involved in a life } \\
\text { situation) }\end{array}$ & $\begin{array}{l}\text { Up to } 1 \text { year } \\
\text { following } \\
\text { hospital } \\
\text { discharge }\end{array}$ \\
\hline \multicolumn{5}{|l|}{ Exclusion criteria } \\
\hline None & \multicolumn{4}{|l|}{$\begin{array}{l}\text { Cancer diagnoses, } \\
\text { neurological pathology or } \\
\text { surgery, cardiovascular } \\
\text { surgery, or pregnancy }\end{array}$} \\
\hline Study type & \multicolumn{4}{|c|}{$\begin{array}{l}\text { Limited to all relevant observational studies (cohort studies and case-control) published in } \\
\text { peer-reviewed journals }\end{array}$} \\
\hline Language & \multicolumn{4}{|l|}{ Limited to English } \\
\hline Year of publication & \multicolumn{4}{|l|}{ No limit } \\
\hline
\end{tabular}

protocol. This systematic review was initiated in December 2014 and is registered with the International Prospective Register of Systematic Reviews (PROSPERO) as 2015:CRD42015023520, available at http://www.crd. york.ac.uk/PROSPERO/. Reporting of the completed systematic review will be according to PRISMA guidelines. Study process, coordination and integrity will be fostered through regular meetings, usually weekly, involving the investigators. Primary communication will be via phone conference supplemented by email communication and face-to-face meetings at annual conferences.

\section{Eligibility criteria}

Study selection criteria were established a priori using a PECOT (population, exposure, comparison, outcomes, time) framework. Study inclusion and exclusion criteria are displayed in table 1 .

\section{Identification and selection of eligible studies}

The search strategy was developed using search terms relevant to the inclusion and exclusion criteria (table 1) and was designed to locate all relevant papers published in peer-reviewed journals. The electronic bibliographic databases to be searched are Medline (Ovid), Cochrane Database of Systematic Reviews (Ovid), Cochrane Central Register of Controlled Trials (Ovid), PubMed, CINAHL (EBSCO), Web of Science and EMBASE. These will be searched from inception. The search strategy will include keywords and database-specific controlled vocabulary relating to critical illness, postintensive care syndrome, intensive care, impairments, activity limitations and participation restrictions. Review articles identified by the search will be obtained and the reference lists of those articles will be examined to reveal additional studies not identified through the search of the electronic bibliographic databases. Language will be restricted to English. The literature search will be rerun before the final analyses are conducted. An example of our search strategy is shown in the online supplementary material. This search strategy will be modified as appropriate for each of the databases we are including in our search.

\section{Study records/data management}

Titles identified through the searches will be exported into an EndNote library where they will be de-duplicated, screened and managed throughout the duration of the systematic review. Full text of the potentially eligible articles will be retrieved and stored in an online repository (Dropbox) for easy accessibility by all team members. A research librarian will conduct the searches, retrieve the full-text articles, manage the DropBox site and maintain the EndNote library throughout the project.

\section{Selection process}

Titles and abstracts of studies identified through the search, and those from references in review articles, will 
be initially screened independently by two reviewers (who will apply the inclusion and exclusion criteria at this stage) to identify any studies that are potentially eligible. Disagreements will be resolved by a third reviewer. Then, the full text of potentially eligible studies will be retrieved and assessed independently by two reviewers, again using the inclusion and exclusion criteria. Disagreement between reviewers regarding the eligibility of a study will be resolved by review from a third member of the team. Study identification and selection will be summarised using a flow chart as recommended by PRISMA.

\section{Data extraction}

Relevant study and outcome data will be extracted from the studies by one reviewer, and extraction accuracy will be verified by a second reviewer. A third reviewer will resolve any discrepancies. All data extractors will be trained in the extraction process and checked for proficiency prior to performing extractions. A standardised, electronic, prepiloted form will be used to record data from the included studies.

Descriptive data extracted from each study will include the following: the country in which the study was conducted, years of subject enrolment, study design/ method, duration of mechanical ventilation, type of ICU, study inclusion and exclusion criteria, reported measures of impairments of body structures and functions, activity limitations, and/or participation restrictions and their outcomes and time frame(s) for measurement.

For the outcomes of interest (table 1) in each study, data will be extracted for the group with critical illness as well as comparison data (eg, data reported for the control group, normative data or retrospective selfreport data). Data will be extracted at 3, 6, 9 and 12 months following critical illness, if available, to capture the trajectory of change in the outcome measures. We expect that outcomes of interest will be continuous and categorical or dichotomous in nature. We will extract point estimates and measures of variability, frequency counts for categorical and dichotomous data as well as relative measures of risk (eg, risk ratios or ORs accompanied by $95 \%$ CIs) as appropriate. Data will be requested from study authors for studies with unreported data, or data represented only in graphical format.

\section{Critical appraisal of included studies}

Included studies will be assessed for strength of evidence using the Oxford Centre for Evidence-based Medicine Levels of Evidence for Symptom Prevalence Studies. The scores range from 1 (systematic reviews) to 5 (expert opinion). ${ }^{28}$ Two reviewers will independently assign a grade to each study based on the described study methodology. Disagreement will be resolved by a third reviewer.
For each included study, the risk of bias will be assessed using the Newcastle-Ottawa Quality Assessment Scale (NOS), a valid and reliable tool designed for assessing methodological quality of non-randomised cohort and case-control studies. ${ }^{29}$ The NOS criteria evaluate the risk of potential bias arising from selection of participants, comparability of study groups and ascertainment of exposure (case-control studies) or outcome of interest (cohort studies). The NOS uses a semiquantitative rating scale resulting in a total score ranging from 0 (lowest quality) to 9 (highest quality) stars. Studies will be scored independently by two reviewers and disagreements in scores will be resolved by review by a third author.

\section{Data synthesis and analysis}

Descriptive characteristics and outcomes of the included studies will be summarised in tables and in narrative format. The outcome(s) extracted from the studies will be categorised within the domains defined in the ICF as:

- impairments (eg, measures of muscle power, pain and so on);

- activity limitations (eg, measures of walking, eating and so on);

- participation restrictions (eg, measures of doing housework, remunerative employment and so on).

The ICF categories provide the framework for identifying the outcomes experienced by the participants while engaged in day-to-day life. ${ }^{26}$ Disability experienced by participants may be attributable to a problem related to impaired body structure and function, activity limitation, participation restriction or an interplay of problems among these domains. Therefore, outcomes will not be prioritised as there is no established or proven hierarchy within these domains for this patient population.

We anticipate that there will be limited scope for meta-analysis due to heterogeneity of patient outcomes across studies. For studies that are sufficiently clinically homogenous enough to allow data pooling (eg, participant characteristics, comparators and outcomes), we will perform meta-analysis using random-effects models with Review Manager (RevMan, V.5.3) statistical software. Dichotomous outcomes will be presented as relative risks with $95 \%$ CIs, while continuous outcomes will be presented as standardised mean differences with $95 \%$ CIs. A $\mathrm{p}$ value of $<0.05$ will indicate statistical significance. We will evaluate heterogeneity using the $\mathrm{I}^{2}$ statistic, interpreted as: $0-40 \%$ might not be important; 30 $60 \%$ may represent moderate heterogeneity; 50-90\% may represent substantial heterogeneity and $75-100 \%$ considerable heterogeneity. ${ }^{30}$ We do not anticipate that the included studies will provide sufficient data for subgroup analyses to be performed.

\section{DISCUSSION}

To improve the quality of rehabilitation services for people with PICS, there is a need for an evidence-based, 
cohesive identification of the physical problems of PICS. That information is needed to inform healthcare providers, survivors with PICS and their family members for a clear and accurate understanding of the types of problems they should anticipate.

Using rigorous systematic review methodology, we will summarise the current evidence evaluating impairments of body functions and structures, activity limitations and participation restrictions in people with PICS during the first year from ICU discharge. This will be the first systematic review in this field, and as such, will increase our understanding about the typical trajectory of physical recovery from critical illness. Our findings will identify the range of physical problems experienced by this patient group, highlighting for clinicians and researchers where best to target assessment and treatment. We expect our findings will provide a foundation on which comprehensive post-ICU rehabilitation can be developed and refined, leading to improved management of PICS.

\section{Author affiliations}

${ }^{1}$ Department of Rehabilitation Science, University at Buffalo, Buffalo, New York, USA

${ }^{2}$ Montante Family Library, D'Youville College, Buffalo, New York, USA

${ }^{3}$ Department of Physiotherapy, The University of Melbourne, Melbourne, Victoria, Australia

${ }^{4}$ Department of Physical Therapy, Mount Saint Mary's University, Los Angeles, California, USA

${ }^{5}$ Physical Therapy Department, Utica College, Utica, New York, USA

Twitter Follow Alan Lee @alanleeDPT

Contributors PJO is the guarantor. PJO, JCS, RSH, ACL and JMS conceived the idea, planned and designed the study protocol. RSH provided expertise for the data extraction, reporting and statistical analyses. JCS provided the search strategy. JMS wrote the first draft and PJO developed the final version after critical feedback from all authors. All authors have approved the final version of the manuscript.

Funding This work was supported by grants from the American Physical Therapy Association and the Academy of Acute Care Physical Therapy.

Disclaimer The sponsors had no part in the development of this protocol. Competing interests None declared.

Provenance and peer review Not commissioned; externally peer reviewed.

Open Access This is an Open Access article distributed in accordance with the Creative Commons Attribution Non Commercial (CC BY-NC 4.0) license, which permits others to distribute, remix, adapt, build upon this work noncommercially, and license their derivative works on different terms, provided the original work is properly cited and the use is non-commercial. See: http:// creativecommons.org/licenses/by-nc/4.0/

\section{REFERENCES}

1. Society of Critical Care Medicine. Critical Care Statistics. June 1 2016. http://www.sccm.org/Communications/Pages/CriticalCareStats. aspx

2. Dick A, Liu H, Zwanziger J, et al. Long-term survival and healthcare utilization outcomes attributable to sepsis and pneumonia. BMC Health Serv Res 2012;12:432.

3. Zambon M, Vincent JL. Mortality rates for patients with acute lung injury/ARDS have decreased over time. Chest 2008;133:1120-7.

4. Fan E, Cheek F, Chlan L, et al. An official American Thoracic Society Clinical Practice guideline: the diagnosis of intensive care unit-acquired weakness in adults. Am J Respir Crit Care Med 2014;190:1437-46.
5. Fan E, Dowdy DW, Colantuoni E, et al. Physical complications in acute lung injury survivors: a two-year longitudinal prospective study. Crit Care Med 2014;42:849-59.

6. Jackson JC, Mitchell N, Hopkins RO. Cognitive functioning, mental health, and quality of life in ICU survivors: an overview. Psychiatr Clin North Am 2015;38:91-104.

7. Hopkins RO, Suchyta MR, Farrer TJ, et al. Improving post-intensive care unit neuropsychiatric outcomes: understanding cognitive effects of physical activity. Am J Respir Crit Care Med 2012;186:1220-8.

8. Porhomayon J, Joude P, Adlparvar G, et al. The impact of high versus low sedation dosing strategy on cognitive dysfunction in survivors of intensive care units: a systematic review and meta-analysis. J Cardiovasc Thorac Res 2015;7:43-8.

9. Dowdy DW, Eid MP, Sedrakyan A, et al. Quality of life in adult survivors of critical illness: a systematic review of the literature. Intensive Care Med 2005;31:611-20.

10. Needham DM, Davidson J, Cohen $\mathrm{H}$, et al. Improving long-term outcomes after discharge from intensive care unit: report from a stakeholders' conference. Crit Care Med 2012;40:502-9.

11. Wilcox ME, Herridge MS. Long-term outcomes in patients surviving acute respiratory distress syndrome. Semin Respir Crit Care Med 2010;31:55-65.

12. Herridge MS, Tansey CM, Matté A, et al. Functional disability 5 years after acute respiratory distress syndrome. $N$ Engl J Med 2011;364:1293-304.

13. Herridge MS, Cheung AM, Tansey CM, et al. One-year outcomes in survivors of the acute respiratory distress syndrome. $N$ Engl J Med 2003;348:683-93.

14. Denehy L, Berney S, Whitburn L, et al. Quantifying physical activity levels of survivors of intensive care: a prospective observational study. Phys Ther 2012;92:1507-17.

15. van der Schaaf M, Beelen A, Dongelmans DA, et al. Poor functional recovery after a critical illness: a longitudinal study. $J$ Rehabil Med 2009:41:1041-8.

16. Choi J, Tate JA, Rogers MA, et al. Depressive symptoms and anxiety in intensive care unit (ICU) survivors after ICU discharge. Heart Lung 2016;45:140-6.

17. Parker AM, Sricharoenchai T, Raparla S, et al. Posttraumatic stress disorder in critical illness survivors: a metaanalysis. Crit Care Med 2015;43:1121-9.

18. Rabiee A, Nikayin S, Hashem MD, et al. Depressive symptoms after critical illness: a systematic review and meta-analysis. Crit Care Med 2016;44:1744-53.

19. Pandharipande PP, Girard TD, Jackson JC, et al. Long-term cognitive impairment after critical illness. $N$ Engl J Med 2013;369:1306-16

20. Flaatten $\mathrm{H}$, Kvåle R. Survival and quality of life 12 years after ICU. A comparison with the general Norwegian population. Intensive Care Med 2001;27:1005-11.

21. Oeyen SG, Vandijck DM, Benoit DD, et al. Quality of life after intensive care: a systematic review of the literature. Crit Care Med 2010;38:2386-400.

22. Turnbull AE, Rabiee A, Davis WE, et al. Outcome measurement in ICU survivorship research from 1970 to 2013: a scoping review of 425 publications. Crit Care Med 2016;44:1267-77.

23. Nordon-Craft A, Schenkman M, Ridgeway K, et al. Physical therapy management and patient outcomes following ICU-acquired weakness: a case series. J Neurol Phys Ther 2011;35:133-40.

24. Elliott D, Denehy L, Berney S, et al. Assessing physical function and activity for survivors of a critical illness: a review of instruments. Aust Crit Care 2011;24:155-66.

25. Tipping CJ, Young PJ, Romero L, et al. A systematic review of measurements of physical function in critically ill adults. Crit Care Resusc 2012;14:302-11.

26. World Health Organization. The International Classification of Functioning, Disability and Health (ICF). Geneva, 2001.

27. Moher D, Shamseer L, Clarke M, et al. Preferred reporting items for systematic review and meta-analysis protocols (PRISMA-P) 2015 statement. Syst Rev 2015;4:1.

28. Centre for Evidence-based Medicine. Oxford Centre for Evidencebased Medicine-Levels of Evidence (March 2009). http://www. cebm.net/oxford-centre-evidence-based-medicine-levels-evidencemarch-2009/

29. Stang A. Critical evaluation of the Newcastle-Ottawa scale for the assessment of the quality of nonrandomized studies in metaanalyses. Eur J Epidemiol 2010;25:603-5.

30. Deeks JJ, Higgins JPT, Altman DG. Analysing data and undertaking meta-analyses. In: Higgins JPT, Green S, eds. Cochrane handbook for systematic reviews of interventions: The Cochrane Collaboration, 2011. 